\title{
LIVRO DIDÁTICO DE ENSINO MÉDIO, GEOMETRIA E A PRESENÇA DAS TECNOLOGIAS
}

\section{High SCHOOL TEXTBOOKS, GEOMETRY AND THE PRESENCE OF TECHNOLOGIES}

\author{
Rúbia Barcelos Amaral-Schio \\ Departamento de Educação Matemática/PPGEM - UNESP - rubia.amaral@ unesp.br
}

\begin{abstract}
RESUMO
O presente texto é fruto de uma pesquisa em andamento, que dentre seus objetivos está a análise da integração das tecnologias aos livros didáticos de Matemática. A pesquisa é de cunho qualitativo e visa instigar a reflexão sobre as contribuições de diferentes recursos didáticos (tecnologias digitais em particular) às práticas dos professores com livros didáticos. Neste artigo o foco é apresentar parte dos resultados, advindos da análise de atividades de cinco coleções de livros didáticos do Ensino Médio no âmbito das propostas de uso das tecnologias no desenvolvimento de conteúdos de Geometria. Os resultados apontam a pouca presença das tecnologias digitais nos livros do Ensino Médio, sendo raras as atividades que apresentam questões de investigação, de exploração visual de figuras geométricas, e de utilização de softwares de geometria dinâmica, que abordam aspectos do dinamismo desse recurso. A maioria delas se limita à realização e conferência de cálculos. Espera-se que haja uma mudança desse quadro, uma vez que desde 2014 Objetos Educacionais Digitais têm sido incorporados aos livros didáticos, com a proposta de haver uma maior integração das tecnologias ao tradicional recurso impresso que o professor usa para desenvolvimento de suas aulas.
\end{abstract}

PAlaVRaS-Chave: Educação Matemática. Software. Calculadora.

\begin{abstract}
This text is a result of a research in progress, guided by the following guiding question: "How can the Mathematics Teaching Laboratory (MTL) and digital technologies contribute to the process of overcoming difficulties in Geometry presented by the students, from their integration to the didactic materials used in the classroom? ". The research is qualitative and aims to contribute to the current scenario of the classroom, in order to instigate reflection on the contributions of the different didactic resources (digital technologies and materials of the MTL especially) to the practices with the materials most used by teachers: textbooks. In this conference, the focus is to present part of the results, derived from the analysis of activities of six collections of textbooks of the high school in the scope of the proposals of use of technologies in the Geometry development. Results show the low presence of digital technologies in high school textbooks, with few research activities, visual exploration of geometric figures, and the use of dynamic geometry software, which address aspects of the dynamism of this resource. Most of them are limited to performing and checking calculations. It is expected that there will be a variance of this framework, since Digital Learning Objects have been incorporated into textbooks, with the proposal of a greater integration of the technologies to the traditional printed resource that the teacher uses to develop their classes.
\end{abstract}

KEYWORDS: Mathematics Education. Software. Calculator. 


\section{INTRODUÇÃO}

Considerando o relevante objetivo da revista de publicar trabalhos na área de Informática na Educação, este texto visa compartilhar resultados de uma pesquisa em andamento. De natureza qualitativa, a pesquisa visa contribuir com o cenário atual da sala de aula, de modo a instigar a reflexão sobre os diferentes recursos didáticos (tecnologias digitais em particular) às práticas dos professores com uso de livros didáticos.

Um recorte foi dado ao estudo, trazendo resultados de uma das fases da pesquisa: a análise da presença das tecnologias nos livros de Matemática do Ensino Médio aprovados pelo Programa Nacional do Livro Didático (PNLD). Para tanto, foram analisadas cinco coleções no âmbito da presença das tecnologias no desenvolvimento da Geometria, conteúdo foco da pesquisa. Considerando o livro didático o principal material de apoio do professor, é relevante analisar a proposta de integração das tecnologias.

\section{SOBRE LIVRO DIDÁTICO, GEOMETRIA E TECNOLOGIAS}

O uso do livro didático é uma prática no Brasil há anos. Programas governamentais foram criados com o objetivo de garantir a qualidade desse material, fornecido aos alunos das escolas públicas gratuitamente. O papel do livro didático é dar suporte ao professor, que tem liberdade para usá-lo à sua maneira, integrando-o, por exemplo, com outras mídias, como computador, internet, vídeo, material concreto, outros livros etc.

Atualmente, o Programa Nacional do Livro Didático (PNLD) é responsável pela análise dos livros didáticos destinados à Educação Básica.

Após a avaliação das obras, o Ministério da Educação (MEC) publica o Guia de Livros Didáticos com resenhas das coleções consideradas aprovadas. O guia é encaminhado às escolas, que escolhem, entre os títulos disponíveis, aqueles que melhor atendem ao seu projeto político pedagógico (BRASIL, 2012, p.2).

No âmbito dos conteúdos matemáticos que compõem essas obras, vale ressaltar que a trajetória da Geometria nos livros didáticos merece um estudo cuidadoso. Em meados das décadas de 80 e 90, esse conteúdo constava no final dos livros, de modo que se os professores não cumprissem todo o conteúdo do livro, os alunos ficavam prejudicados, pois não era estudado. Do ponto de vista histórico, a análise de Gouvêa (1998, p.43) aponta que, no período pós Matemática Moderna,

O ensino da Geometria passou a ser abandonado pelos professores, os quais a planejavam para o último ano [...]. Ensinar e aprender Geometria por meio de espaços vetoriais ou por meio de transformações, como pregava a Matemática Moderna, era difícil tanto para professores, como para alunos, por se tratar de nova abordagem. E a Geometria, cada dia mais, foi sendo relegada ao último plano do currículo escolar de $1^{\circ}$. grau.

Em consequência desse caminhar histórico, a Geometria é um tema considerado problemático pelos professores, que costumam ter dificuldade, tanto com seus conceitos como com seu ensino (ALMOULOUD et al., 2004). Costa (2008, p.32) afirma que "grande parte dos alunos ao saírem da educação básica, principalmente da escola pública, não têm os conhecimentos básicos de Geometria esperados para esse nível de ensino". Os Parâmetros Curriculares Nacionais (PCN) há anos já ressaltava que a Geometria costuma receber pouco destaque nas aulas de Matemática e, muitas vezes, é confundida com o ensino de medidas, apesar de desempenhar papel fundamental no currículo, por desenvolver um tipo particular de pensamento para compreender, descrever e representar, de forma organizada, o mundo em que vive o aluno. Atualmente, a Base Nacional Comum Curricular (BNCC) observa que "a Geometria não pode ficar reduzida a mera aplicação de fórmulas de cálculo de área e de volume nem a aplicações numéricas imediatas de 
teoremas sobre relações de proporcionalidade em situações relativas a feixes de retas paralelas cortadas por retas secantes ou do teorema de Pitágoras" (BRASIL, 2018, p.272).

Nessa direção, pode-se considerar que estava se constituindo um "ciclo vicioso", pois com pouco estudo destinado à Geometria nas aulas de Matemática, ao longo da Educação Básica, o professor, então aluno, se formava com deficiência nesse conteúdo, nem sempre suprida pelo Ensino Superior. Quando egresso de seu curso de formação, em suas aulas pouco aprofundava o tema com seus alunos. Almouloud et al. (2004, p.99) apontam, "em relação à formação dos professores, que esta é muito precária quando se trata de geometria, pois os cursos de formação inicial não contribuem para que [os futuros professores] façam uma reflexão mais profunda a respeito do ensino e da aprendizagem dessa área da matemática".

No âmbito da aprendizagem dos alunos em Geometria,

A avaliação educacional da rede estadual de São Paulo em 1998 - Sistema de Avaliação de Rendimento Escolar do Estado de São Paulo (SARESP, 2000) revela que muitos tópicos de matemática, pelo fato de não serem planejados ou ensinados pelos professores, não são aprendidos por seus alunos. Um exemplo disso é que, embora os professores indiquem a geometria como item importante, que merece lugar em todos os níveis de ensino, não há concordância quanto à seleção e à organização dos conteúdos a serem ensinados tanto no ensino fundamental como no ensino médio (ALMOULOUD et al., 2004, p.94-95).

Há alguns anos, no entanto, tem havido ações para reverter essa realidade. A Geometria agora não mais é deixada para o final em muitos livros, mas está presente logo no início, ou se intercalando com os demais temas (GODOY, 2016). Ademais, desde a ampliação da divulgação dos softwares de geometria dinâmica, mais fortemente a partir de meados do ano 2000, muitas têm sido as pesquisas que apontam suas potencialidades para os processos de ensino e aprendizagem de Geometria, dando novo "status" a ela. A atual BNCC recomenda fortemente o uso desse tipo de software (BRASIL, 2018).

O Governo do Estado de São Paulo, por exemplo, comprou a licença do CabriGeometre, há mais de quinze anos, para todas as suas escolas públicas e ofereceu cursos de capacitação aos professores. No entanto, o que se percebeu é que os softwares não eram muito utilizados nas aulas de Matemática (ALMOULOUD et al., 2004). O software GeoGebra, que é gratuito, se expandiu pelo mundo especialmente nos últimos anos, disponibilizando muitos materiais em seu sítio oficial ${ }^{1}$ para os professores, e também muitos cursos têm sido oferecidos, inclusive pelo Governo Estadual em São Paulo. Não obstante, os computadores das escolas públicas estaduais envolvidas no Programa Acessa Escola já contam com o Geogebra instalado. Ainda assim, continuam sendo poucas as experiências efetivas de utilização de softwares de geometria dinâmica na sala de aula (SARTI, 2012), fato que não se restringe ao Estado de São Paulo.

$\mathrm{O}$ uso de outras tecnologias, como a calculadora, também tem sido foco de estudo no âmbito dos livros didáticos (como por Borba e Selva (2013)). Pesquisas têm mostrado que a presença das tecnologias em atividades propostas em livros didáticos podem influenciar positivamente o aprendizado dos alunos e, em particular, seu desenvolvimento matemático (DUFFIN, 1997; GROVES, 1994; MEDEIROS, 2003, SELVA; BORBA, 2010; BORBA; SELVA, 2013). Nessa direção, há anos se defende fortemente que, no ensino de Matemática, recursos contemporâneos como computadores e calculadoras devem estar integrados à prática pedagógica (por exemplo, Noss e Hoyles (1996), Artigue (1997) e Borba e Penteado (2005)), como também já recomendava os PCN e, atualmente, a Base Nacional Curricular Comum (BNCC).

${ }^{1}$ Disponível em:<www.geogebra.org>. Acesso em 21 out. 2013. 


\section{Procedimentos metodológicos}

Como mencionado anteriormente, a pesquisa tem sido desenvolvida assumindo uma abordagem interpretativa de pesquisa qualitativa. Como afirmam Bogdan e Biklen (1994, p.209), os estudos dessa natureza "devem revelar maior preocupação pelo processo e significado e não pelas suas causas e efeitos". E, nesse sentido, o foco está na integração das tecnologias digitais às aulas de Geometria, em interlocução com os materiais didáticos (livros didáticos), e não em seus resultados ou produtos, o que contempla uma das características centrais da pesquisa qualitativa, segundo Denzin e Lincoln (2000).

Vale ressaltar ainda, segundo Alves-Mazzotti (2001, p.131), que "a principal característica das pesquisas qualitativas é o fato de que estas seguem uma tradição 'compreensiva' ou interpretativa". Além disso, Bogdan e Biklen (1994) orientam a estudar objetivamente as questões subjetivas inerentes à pesquisa.

Os resultados aqui apresentados são fruto de uma das fases da pesquisa: a análise da presença das tecnologias nas atividades propostas em livros didáticos de Matemática do Ensino Médio. Esse processo se iniciou pelas coleções aprovadas pelo PNLD 2015, pois os livros desse ano eram os que estavam em vigência nas escolas. Conforme os livros do PNLD 2018 têm sido adquiridos ${ }^{2}$, os dados têm sido atualizados. Cabe salientar que ao comparar as coleções que se teve acesso, é possível notar que há poucas diferenças entre as edições de 2015 e 2018, de modo que os resultados aqui descritos são representativos dos materiais que estão em sala de aula atualmente. Nesse cenário, é apresentada aqui a análise de cinco coleções, aqui denominadas de Livro 1, Livro 2, etc., sendo os três primeiros os mais recentes.

Essa primeira fase dá suporte à pesquisa na medida em que retrata a integração das tecnologias ao principal material de apoio do professor, levando à reflexão sobre como as mídias se fazem presentes nos livros didáticos, e o papel do professor nesse cenário.

\section{A PRESENÇA DAS TECNOLOGIAS NOS LIVROS DIDÁtiCOS DE MATEMÁtiCA}

Os professores supostamente têm escolhido ${ }^{3}$ os livros a serem usados em suas aulas a partir das resenhas apresentadas no Guia do Livro Didático (BRASIL, 2017). Este documento, assim como as referências mencionadas anteriormente, ressaltam o papel das tecnologias: "o Ensino Médio tem de assumir a tarefa de preparar cidadãos para uma sociedade cada vez mais permeada por novas tecnologias e de possibilitar o ingresso de parcelas significativas de seus cidadãos a patamares mais elaborados do saber" (BRASIL, 2017, p.11).

Ao analisar os livros didáticos, especialmente as atividades, é possível obter um retrato que pouco está em sintonia com o que tem apontado a literatura e os documentos oficiais. O Quadro 1 descreve a quantidade de atividades presente nos capítulos que contemplam os conteúdos de Geometria Plana e Espacial, como também a quantidade de propostas relacionadas à utilização do computador (como aplicativos em sítios, conteúdos digitais e softwares) e da calculadora. Para tanto, as atividades foram contabilizadas

\footnotetext{
${ }^{2}$ Como os livros aprovados pelo PNLD não são comercializados, o acesso a eles não é tão simples, pois advêm de doação de escolas, de Diretorias de Ensino, etc.

${ }^{3} \mathrm{Na}$ prática há muitos relatos de que as escolas recebem alguns livros e que os professores consideram esse contato para observar o conteúdo dos livros e, então, fazerem a escolha. Os Editais de 2019 e 2020 determinam que as editoras disponibilizem um link para os professores acessarem os conteúdos (ou parte deles) de todas as coleções aprovadas, ainda que também contemplem a elaboração do Guia para subsidiar a escolha dos professores.
} 
considerando também os seus subitens como unidade, uma vez que podem ser identificadas individualmente como um exercício proposto (veja a figura 1, por exemplo. Cada um dos subitens pede o cálculo do volume de um sólido geométrico diferente. Portanto, ainda que aglutinados em um mesmo exercício, com enunciado comum, o aluno encontra uma solução e uma resposta para cada um dos subitens).

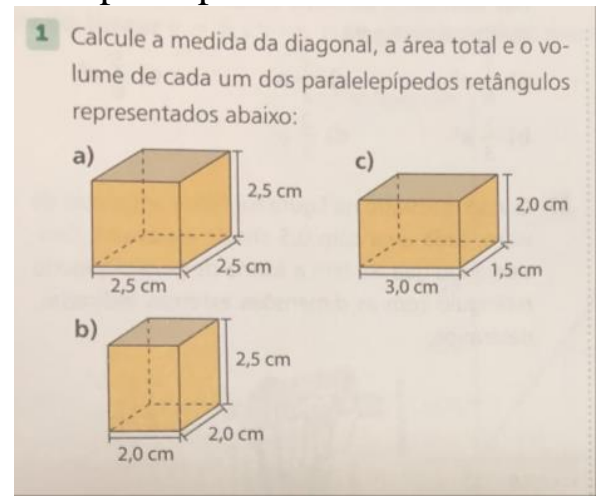

Figura 1 - Exercício com três subitens Fonte: Livro 3 (IEZZI et al., 2016, p.157)

Quadro 1: Quantidade de atividades envolvendo tecnologias

\begin{tabular}{|c|c|c|c|c|c|}
\hline \multirow[t]{2}{*}{ Livro } & \multirow[t]{2}{*}{ Ano } & \multirow[t]{2}{*}{ Título do capítulo/ unidade } & \multirow{2}{*}{$\begin{array}{c}\text { Quantidade } \\
\text { de } \\
\text { exercícios }\end{array}$} & \multicolumn{2}{|c|}{$\begin{array}{c}\text { Quantidade de exercícios } \\
\text { com tecnologia }\end{array}$} \\
\hline & & & & Computador & Calculadora \\
\hline \multirow{4}{*}{$\begin{array}{l}\text { Livro 1 } \\
\text { (PNLD } \\
2018)\end{array}$} & $2^{\circ}$ & $\begin{array}{l}\text { Capítulo } 8 \text { - Área de figuras } \\
\text { planas }\end{array}$ & 47 & 1 & - \\
\hline & \multirow[t]{3}{*}{$3^{\circ}$} & $\begin{array}{l}\text { Capítulo } 1 \text { - Geometria espacial e } \\
\text { de posição }\end{array}$ & 99 & - & - \\
\hline & & Capítulo 2 - Poliedros & 53 & - & - \\
\hline & & Capítulo 3 - Corpos redondos & 77 & - & - \\
\hline \multirow{5}{*}{$\begin{array}{l}\text { Livro } 2 \\
\text { (PNLD } \\
2018)\end{array}$} & \multirow[t]{2}{*}{$1^{\circ}$} & $\begin{array}{l}\text { Capítulo } 10 \text { - Semelhança e } \\
\text { triângulos retângulos }\end{array}$ & 67 & - & - \\
\hline & & $\begin{array}{l}\text { Capítulo } 12 \text { - Área de figuras } \\
\text { planas }\end{array}$ & 105 & - & - \\
\hline & \multirow[t]{3}{*}{$2^{\circ}$} & $\begin{array}{l}\text { Capítulo } 7 \text { - Geometria espacial } \\
\text { de posição }\end{array}$ & 118 & - & - \\
\hline & & Capítulo 8 - Poliedros & 107 & - & - \\
\hline & & Capítulo 9 - Corpos redondos & 119 & - & - \\
\hline \multirow{5}{*}{$\begin{array}{l}\text { Livro } 3 \\
\text { (PNLD } \\
2018)\end{array}$} & $1^{\circ}$ & $\begin{array}{l}\text { Capítulo } 10 \text { - A semelhança e os } \\
\text { triângulos }\end{array}$ & 67 & 1 & - \\
\hline & \multirow{4}{*}{$2^{\circ}$} & $\begin{array}{l}\text { Capítulo } 4 \text { - Superfícies } \\
\text { poligonais, círculos e áreas }\end{array}$ & 50 & - & - \\
\hline & & $\begin{array}{l}\text { Capítulo } 5 \text { - Introdução à } \\
\text { Geometria espacial }\end{array}$ & 64 & - & - \\
\hline & & Capítulo 6 - Poliedros & 127 & - & - \\
\hline & & Capítulo 7 - Corpos redondos & 94 & - & - \\
\hline \multirow{3}{*}{$\begin{array}{l}\text { Livro } 4 \\
\text { (PNLD } \\
2015)\end{array}$} & \multirow[t]{3}{*}{$2^{\circ}$} & $\begin{array}{l}\text { Unidade } 7 \text { - Sólidos geométricos: } \\
\text { poliedros }\end{array}$ & 111 & 1 & - \\
\hline & & $\begin{array}{l}\text { Unidade } 8 \text { - Sólidos geométricos: } \\
\text { corpos redondos }\end{array}$ & 36 & 1 & - \\
\hline & & $\begin{array}{l}\text { Unidade } 9 \text { - Geometria métrica } \\
\text { espacial }\end{array}$ & 271 & - & 4 \\
\hline \multirow[b]{2}{*}{$\begin{array}{l}\text { Livro } 5 \\
\text { (PNLD } \\
\text { 2015) }\end{array}$} & \multirow[b]{2}{*}{$2^{\circ}$} & $\begin{array}{l}\text { Capítulo } 7 \text { - Polígonos inscritos e } \\
\text { áreas }\end{array}$ & 72 & 2 & - \\
\hline & & $\begin{array}{l}\text { Capítulo } 8 \text { - Geometria espacial } \\
\text { de posição: uma abordagem } \\
\text { intuitiva }\end{array}$ & 142 & 1 & - \\
\hline
\end{tabular}




\begin{tabular}{|l|l|l|c|c|}
\hline & $\begin{array}{l}\text { Capítulo 9 - Poliedros: prismas e } \\
\text { pirâmides }\end{array}$ & 78 & 2 & 3 \\
\cline { 2 - 5 } & Capítulo 10 - Corpos redondos & 68 & 1 & 2 \\
\hline
\end{tabular}

Fonte: Elaborado pela autora

Ao analisar este quadro, o principal resultado, de simples visualização, é a "baixa presença" das tecnologias nos livros didáticos do Ensino Médio. O Guia também ressalta esse fato, especialmente no âmbito da Geometria:

Quanto aos recursos didáticos, o uso de ferramentas tecnológicas ainda é um terreno pouco explorado no Ensino Médio atual. Por exemplo, nas obras analisadas, o emprego da calculadora é frequente, porém comumente voltado para a realização e a conferência de cálculos, em detrimento de outras possibilidades de trabalho.

Entre os outros recursos tecnológicos, de forma geral, há boas sugestões de utilização de softwares livres. Contudo, na maioria das obras, raramente é destacado o uso de instrumentos de desenho na aprendizagem de conceitos geométricos (BRASIL, 2017, p.40).

Cabe ressaltar que os nove livros das três primeiras coleções não apresentam nenhuma atividade de Geometria Espacial com integração das tecnologias. Destaca-se, ainda, que a única atividade de Geometria Plana do Livro 1 não indica, de fato, o papel do recurso tecnológico (figura 2). No início do livro é indicado o símbolo que aparece em atividades que envolvem tecnologia (figura 3), sendo este presente no exercício 3, mas não está claro como o computador estaria sendo usado para sua resolução, ainda mais considerando que o enunciado solicita o cálculo no caderno.

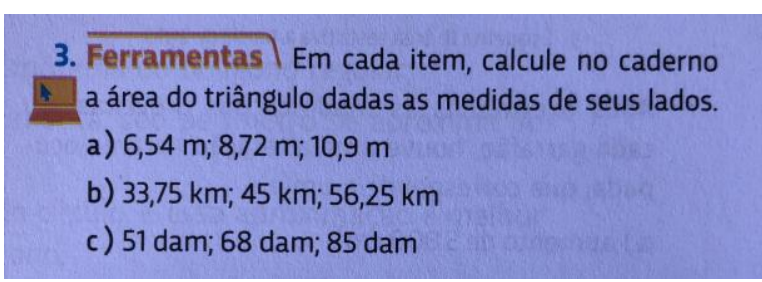

Figura 3 - Exercício com indicação de tecnologia

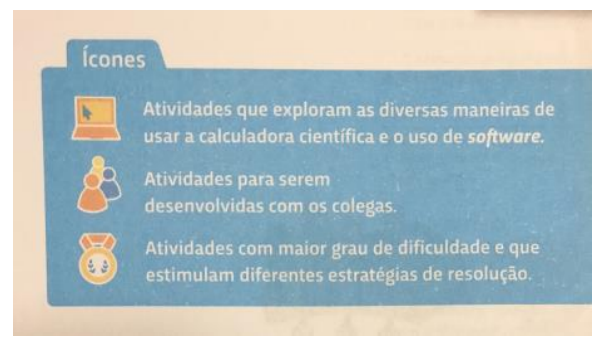

Figura 2 - Símbolo de tecnologia

Fonte: Livro 1 (CHAVANTE; PRESTES, 2016, p.201 à esquerda e p.4 à direita)

No Livro 3, por sua vez, a única indicação do uso da tecnologia não aparece em meio às atividades para os alunos, ou seja, para ser desenvolvida por estes. Certamente é relevante o fato de haver pelo menos uma sugestão de uso do computador (figuras 4), mas não se pode deixar de ressaltar que não é proposto como parte das atividades em que efetivamente os alunos têm papel ativo diante das tecnologias. 
Figura 4 - Proposta de utilização de software de geometria dinâmica Fonte: Livro 3 (LEONARDO, 2016, p.225-226)

Duas das coleções analisadas contêm propostas de utilização da calculadora nas atividades. Por ser um recurso associado às quatro operações básicas, a calculadora aparece indicada essencialmente para o desenvolvimento de cálculos, como volume e área de figuras geométricas (as figuras 5 e 6 contêm exemplos), como bem descreve o Guia (BRASIL, 2017). 


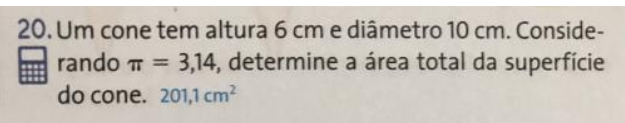

Figura 6 - Atividades com sugestão de uso da calculadora Fonte: Livro 5 (DANTE, 2013, p.225)

Quanto às propostas com computador, o Livro 5 apresenta 6 ícones que indicam "conteúdo digital", supostamente relacionado ao conteúdo em estudo. No entanto, não há nenhuma descrição desse recurso, de modo que os ícones aparecem como uma etiqueta colada em qualquer local da página do livro (ver exemplo na figura 7), como discutem Ribeiro e Amaral (2016) ao tratar desse tipo de recurso em livros didáticos aprovados pelo PNLD.

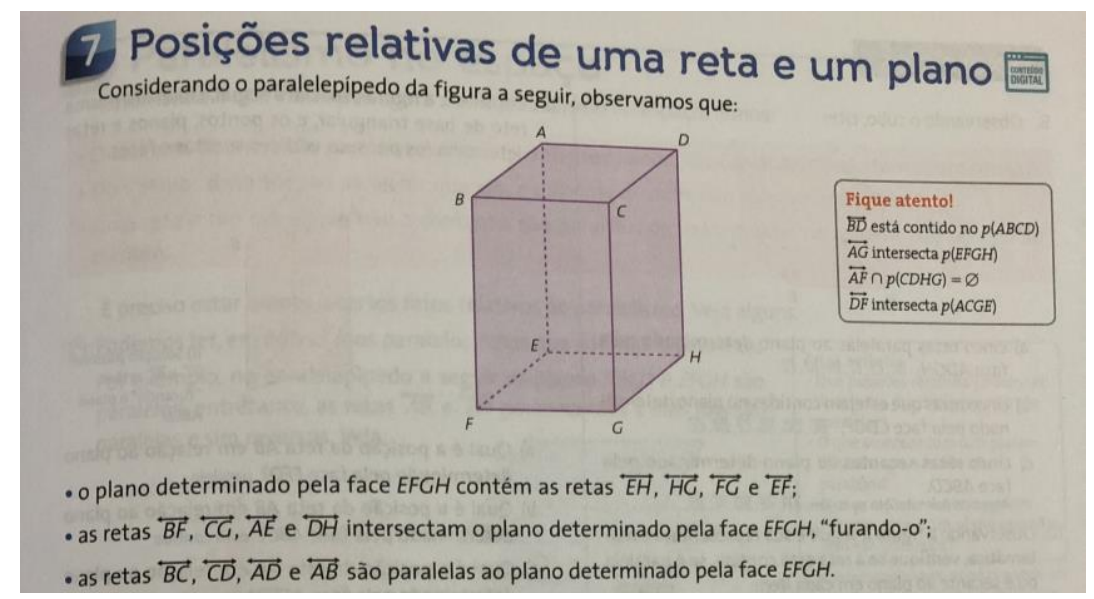

Figura 7 - Página do livro que contém um ícone indicando "conteúdo digital" Fonte: Livro 5 (DANTE, 2013, p.167)

O Livro 4 apresenta duas propostas de utilização de recursos, especificamente o uso de aplicativos em sítios (figura 8 é uma das atividades). Assim como o Livro 1, há um ícone que destaca a presença de uma atividade com uso do computador. Vale salientar que o mesmo se observa nos Livros 4 e 5 no que tange às atividades com calculadoras.

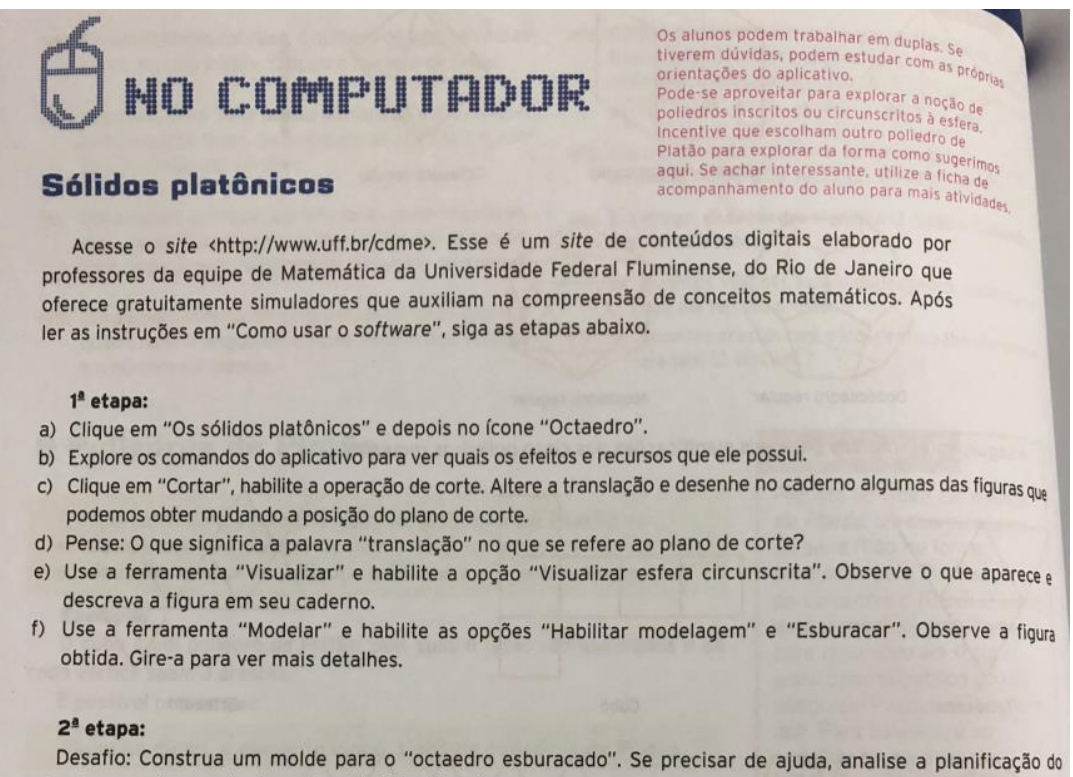


Figura 7 - Atividade com uso de computador

Fonte: Livro 4 (SMOLE; DINIZ, 2013, p.166)

O quadro e as figuras acima ilustram a presença das tecnologias nos livros didáticos do Ensino Médio de forma muito aquém do que recomendam há anos os documentos oficiais, reforçado pelo Guia (BRASIL, 2017), dando indicativo de que esse tema merece atenção dos autores e editores. Nesse contexto, os resultados dessa primeira fase da pesquisa levam à observação de que são raras as atividades que apresentam potencialidades do uso das tecnologias em sala de aula, como investigação, de exploração visual de figuras geométricas, e de utilização de softwares de geometria dinâmica, que abordam aspectos do dinamismo desse recurso. A maioria delas se limita à realização e conferência de cálculos. Esse cenário sugere a reflexão de que, atualmente, cabe ao professor ampliar as possibilidades de integração das tecnologias aos livros didáticos, de modo que a pesquisa tem se organizado para promover meios para isso, na expectativa de que haja uma mudança desse quadro.

\section{CONSIDERAÇõES FINAIS}

Diante do contexto da pesquisa em andamento que originou a análise aqui apresentada, se fez relevante estudar as atividades propostas com uso da calculadora e de recursos disponíveis no computador ${ }^{4}$ (como softwares, conteúdos digitais e aplicativos), uma vez que um dos objetivos é a integração da tecnologia às aulas de Matemática, considerando a conexão com os livros didáticos, visto que esse é o recurso mais utilizado pelos professores no planejamento de suas aulas.

Desde 2014 Objetos Educacionais Digitais têm sido incorporados aos livros didáticos aprovados pelos PNLD, o que sugere que a integração das tecnologias aos livros didáticos venha sendo fomentada por essa iniciativa. Desse modo, vislumbrava-se apresentar resultados semelhantes ao que Borba e Selva (2013) descrevem no âmbito das atividades propostas com calculadoras nos livros aprovados para os Anos Iniciais do Ensino Fundamental, discutindo quais as diferentes abordagens contidas nas atividades, e sua contribuição para os processos de ensino e aprendizagem da Matemática.

No entanto, o que se constatou foi que nos livros destinados ao Ensino Médio, contrariamente ao que recomendam os documentos oficiais, e o relevante papel que indicam as pesquisas em Educação Matemática, a tecnologia pouco se faz presente. A calculadora se limita à resolução das quatro operações básicas (o que de certo modo é natural, já que as atividades analisadas tangem conceitos de Geometria). O uso do computador, por sua vez, é sugerido em poucas atividades: em dois livros aparecem efetivamente interligado às atividades; e em um deles há apenas uma "etiqueta" que menciona conteúdo digital supostamente relacionado ao tema.

Espera-se que o retrato aqui apresentado leve à reflexão dos autores de livros didáticos, de modo a reestruturarem suas próximas edições; bem como de professores de Matemática, de modo que identifiquem que, por enquanto, é preciso encontrar outros meios de integração da tecnologia na sala de aula que não através de atividades propostas nos livros frequentemente utilizados em suas aulas.

\section{REFERÊNCIAS BIBLIOGRÁFICAS}

\footnotetext{
${ }^{4}$ Hoje já diversos desses recursos disponíveis para dispositivos móveis. Seguindo as indicações dos livros, optou-se por restringir ao uso desses recursos por meio dos computadores.
} 
ALMOULOUD, S. A. et al. A geometria no ensino fundamental: reflexões sobre uma experiência de formação envolvendo professores e alunos. Revista Brasileira de Educação, Rio de Janeiro, n.27, p.94-108, set./dez. 2004.

ALVES-MAZZOTTI, A.J. O método nas Ciências Sociais. In: ALVES-MAZZOTTI, A.J.; GEWANDSZNAJDER, F. O método nas Ciências Naturais e Sociais: Pesquisa Quantitativa e Qualitativa. São Paulo: Editora Pioneira, 2001. p.107-188.

ARTIGUE, M. Computer environments and learning theories in mathematics education. In: BARZEL, B. (Ed.), Teaching Mathematics with Derive and the TI-92. Proceedings of the International Derive and TI-92 Conference, 1997, p. 1-17.

BOGDAN, R. C.; BIKLEN, S. K. Investigação qualitativa em educação. Porto: Porto Editora, 1994.

BORBA, M.C.; PENTEADO, M.G. Informática e educação matemática. Belo Horizonte: Autêntica, 2005.

BORBA, R.; SELVA, A. Analysis of the role of the calculator in Brazilian textbooks. ZDM Mathematics Education. n.45, p.737-750, 2013.

BRASIL. Ministério da Educação. Base Nacional Comum Curricular (BNCC). Disponível em: http://basenacionalcomum.mec.gov.br/wpcontent/uploads/2018/12/BNCC_14dez2018_site.pdf. Acesso em: 19 dez. 2018.

BRASIL. Ministério da Educação. Programa Nacional do Livro Didático (PNLD). Disponível em:http://portal.mec.gov.br/index.php?Itemid=668\&id=12391\&option= com_content\&view=article>. Acesso em: 20 out. 2012.

BRASIL. Guia de livros didáticos. PNLD 2018: Matemática. Ensino Médio. Secretária de Educação Básica - SEB - Brasília, DF: Ministério da Educação, 2017.

CHAVANTE, E.; PRESTES, D. Quadrante. 1.ed. São Paulo: Edições SM, 2016.

DANTE, L.R. Matemática: contexto \& aplicações. 2.ed. São Paulo: Ática, 2013.

DENZIN, N.K.; LINCOLN, Y.S. The discipline and practice of qualitative research, In: DENZIN, N.; LINCOLN, Y.S. Handbook of qualitative research. Londres: Sage, 2000.

DUFFIN, J. The role of calculators. In: Thompson, I. (Ed.).Teaching and learning early numbers. UK: Open University Press, 1997.

GODOY, J.S. A Geometria presente em alguns livros didáticos do Ensino Fundamental. 2016. Dissertação (Mestrado em Educação Matemática) - Universidade Estadual Paulista, 2016.

GOUVÊA, F. A. T. Aprendendo e ensinando Geometria com a demonstração: uma contribuição para a prática pedagógica do professor de Matemática do Ensino Fundamental. 1998. Dissertação (Mestrado em Educação Matemática)-Pontifícia Universidade Católica de São Paulo, São Paulo,1998.

GROVES, S. The effect of calculator use on third and fourth graders' computation and choice of calculating device. In: Proceedings of the 18th International Conference for the Psychology of Mathematics Education, PME 18, vol. 3. Lisboa, 1994.

IEZZI, G.; DOLCE, O.; DEGENSZAJN, D.; PÉRIGO, R.; ALMEIDA, N. Matemática: ciência e aplicações. Ensino Médio, $2^{\circ}$ ano. 9.ed. São Paulo: Saraiva: 2016.

LEONARDO, F.M. Conexões com a Matemática. 3.ed. São Paulo: Moderna, 2016. MEDEIROS, K. A influência da calculadora na resolução de problemas matemáticos abertos. Educação Matemática em Revista, 14. São Paulo: SBEM, 2003.

NOSS, R.; HOYLES, C. Windows on mathematical meanings.Dordrecht: Kluwer, 1996. 
RIBEIRO, M; Amaral, R.B. Guia e tecnologia dos/nos livros didáticos de Matemática: uma primeira discussão. Educação Matemática em Revista. v.51, 2016. 2016, p.64-75. SELVA, A.; BORBA, R. O uso da calculadora nos anos iniciais do Ensino Fundamental. Belo Horizonte: Autêntica, 2010.

SMOLE, K.; DINIZ, M.I. Matemática Ensino Médio. 8.ed. São Paulo: Saraiva, 2013. 